\title{
Assessing spacing impact on coherent features in a wind turbine array boundary layer
}

\author{
Naseem Ali ${ }^{1}$, Nicholas Hamilton ${ }^{2}$, Dominic DeLucia ${ }^{1}$, and Raúl Bayoán Cal ${ }^{1}$ \\ ${ }^{1}$ Department of Mechanical and Materials Engineering, Portland State University, Portland, Oregon, USA \\ ${ }^{2}$ National Renewable Energy Laboratory, Boulder, Colorado 80401, USA \\ Correspondence: Raúl Bayoán Cal (rcal@pdx.edu)
}

Received: 20 July 2017 - Discussion started: 7 August 2017

Revised: 17 December 2017 - Accepted: 8 January 2018 - Published: 28 February 2018

\begin{abstract}
As wind farms become larger, the spacing between turbines becomes a significant design consideration that can impose serious economic constraints. To investigate the turbulent flow structures in a $4 \times 3$ Cartesian wind turbine array boundary layer (WTABL), a wind tunnel experiment was carried out parameterizing the streamwise and spanwise wind turbine spacing. Four cases are chosen spacing turbines by 6 or $3 D$ in the streamwise direction, and 3 or $1.5 D$ in the spanwise direction, where $D=12 \mathrm{~cm}$ is the rotor diameter. Data are obtained experimentally using stereo particle image velocimetry. Mean streamwise velocity showed maximum values upstream of the turbine with the spacing of 6 and $3 D$ in the streamwise and spanwise direction, respectively. Fixing the spanwise turbine spacing to $3 D$, variations in the streamwise spacing influence the turbulent flow structure and the power available to following wind turbines. Quantitative comparisons are made through spatial averaging, shifting measurement data and interpolating to account for the full range between devices to obtain data independent of array spacing. The largest averaged Reynolds stress is seen in cases with spacing of $3 D \times 3 D$. Snapshot proper orthogonal decomposition (POD) was employed to identify the flow structures based on the turbulence kinetic energy content. The maximum turbulence kinetic energy content in the first POD mode is seen for turbine spacing of $6 \mathrm{D} \times 1.5 \mathrm{D}$. The flow upstream of each wind turbine converges faster than the flow downstream according to accumulation of turbulence kinetic energy by POD modes, regardless of spacing. The streamwise-averaged profile of the Reynolds stress is reconstructed using a specific number of modes for each case; the case of $6 D \times 1.5 D$ spacing shows the fastest reconstruction to compare the rate of reconstruction of statistical profiles. Intermediate modes are also used to reconstruct the averaged profile and show that the intermediate scales are responsible for features seen in the original profile. The variation in streamwise and spanwise spacing leads to changes in the background structure of the turbulence, where the color map based on barycentric map and Reynolds stress anisotropy tensor provides an alternate perspective on the nature of the perturbations within the wind turbine array. The impact of the streamwise and spanwise spacings on power produced is quantified, where the maximum production corresponds with the case of greatest turbine spacing.
\end{abstract}

\section{Introduction}

Allowing insufficient space between wind turbines in an array leads to decreased performance through wake interaction, decreased bulk flow velocity, and an increase in the accumulated fatigue loads and intermittency events on downstream turbines (Viggiano et al., 2016; Ali et al., 2016a). Wind turbine wakes lead to an average loss of $10-20 \%$ of the total potential power output of wind turbine array (Barthelmie et al.,
2007). Extensive experimental and numerical studies focus on wake properties in terms of the mean flow characteristics used to obtain estimates of power production (Chamorro and Porté-Agel, 2009, 2011). Wake growth depends on the shape and magnitude of the velocity deficit, surface roughness, flow above the canopy, and spacing between the turbines.

Although there are many studies dealing with the effect of the density of turbines on the wake recovery, it is still a de- 
bated question. The actual spacing of wind turbines can vary greatly from one array to another and depending on the direction of the bulk flow. For example, in the Nysted farm, spacing is 10.5 diameters $(D)$ downstream by $5.8 D$ spanwise at the exact row (ER). The wind direction at the ER is $278^{\circ}$ and mean wind direction can deviate from ER by $\pm 15^{\circ}$ (Barthelmie et al., 2010). Variation in the wind direction is evident through wake statistics, including wake width, center line, and orientation with respect to the array. Barthelmie and Jensen (2010) showed that the spacing in the Nysted farm is responsible for $68-76 \%$ of the farm efficiency variation. In the Horns Rev farm, spacing between devices is $7 D$, although aligned with the bulk flow direction spacing is as much as 10.4D. Hansen et al. (2012) pointed out that variations in the power deficit are almost negligible when spacing is approximately $10 \mathrm{D}$ at the Horns Rev farm, in contrast to limited spacings that present a considerable power deficit. González-Longatt et al. (2012) found that when the streamwise and spanwise spacing increased, the wake coefficient, which represents the ratio of total power output with and without wake effects, is increased. Nilsson et al. (2015) performed large-eddy simulations (LESs) of the Lillgrund wind farm, where pre-generated turbulence and wind shear are imposed in the computational domain to simulate realistic atmospheric conditions. In the Lillgrund wind farm, the actual spacing is 3.3 and $4.6 \mathrm{D}$ in the streamwise and spanwise directions. A turbine is missing near to the center of the wind farm, demonstrating the effects of a farm with limited spacing and one with sufficient spacing in otherwise identical operating conditions. The results of Nilsson et al. (2015) are highly applicable in the current study, although their foci are on turbulence intensity effects and yaw angle.

Further, the effect of the incoming flow direction on the wake coefficient increased when the spacing of the array was reduced. Meyers and Meneveau (2012) studied the optimal spacing in a fully developed wind farm under neutral stratification and flat terrain. The results highlighted that, depending on the ratio of land and turbine costs, the optimal spacing might be $15 D$ instead of $7 D$. Stevens (2015) pronounced that the optimal spacing depends on the length of the wind farm in addition to the factors suggested in Meyers and Meneveau (2012). Orography and wind direction are relevant when deciding distance between turbines as well as layout, as shown by Romanic et al. (2018).

Further investigations in array optimization have been undertaken by changing the alignment of the wind farm, often referred to as staggered wind farms. Meyers and Meneveau (2010) compared aligned versus staggered wind farms, the latter yielding a $5 \%$ increase in extracted power. Yang et al. (2012) used LES to study the influence of the streamwise and spanwise spacing on the power output in aligned wind farms under a fully developed regime. Their work confirmed that power produced by the turbines scales with streamwise spacing more than with the spanwise spacing. Wu and PortéAgel (2013) investigated turbulent flow within and above aligned and staggered wind farms under neutral conditions. Cumulative wakes are shown to be subject to strong lateral interaction in the staggered case. In contrast, lateral interaction is negligible in the aligned wind farm. Archer et al. (2013) quantified the influence of wind farm layout on the power production, verifying that increasing the turbine spacing in the predominant wind direction maximized the power production, regardless of device arrangement in the wind farm. Stevens et al. (2016) investigated the power output and wake effects in aligned and staggered wind farms with different streamwise and spanwise turbine spacings. In the staggered configuration, power output in a fully developed flow depends mainly on the spanwise and streamwise spacings, whereas in the aligned configuration, power strongly depends on the streamwise spacing.

As wind farms become larger, the land costs and availability represent critical factors in the overall value of the wind farm. Spacing between the turbines is an important design factor in terms of overall wind farm performance and economic constraints. Investigation of wind farms with limited spacing is important in order to quantify the effects of wind turbine wake interaction on the power production. The current work compares the turbulent flow in various configurations of the array, where the streamwise and spanwise spacings are varied. The tunnel-scaled wind farm is restricted to a flat surface and topographic influences are not considered, although the inflow to the wind farm includes modifications to resemble an atmospheric boundary layer. The performance of the arrays is characterized by analyzing the mean velocity, Reynolds shear stress, and power production. Proper orthogonal decomposition (POD) is employed to identify coherent structures of the turbulent wake associated with variations in spacing. The Reynolds stresses are reconstructed from a POD basis, demonstrating variation in rates of convergence according to wind turbine spacing. Finally, the Reynolds stress anisotropy tensor is employed to differentiate the balance of energy in the turbulence field for the test cases.

\section{Theory}

\subsection{Snapshot proper orthogonal decomposition}

POD is a mathematical tool that derives optimal basis functions from a set of measurements, decomposing the flow into modes that express the most dominant features. The technique, which was presented in the frame of turbulence by Lumley (1967), categorizes structures within the turbulent flow depending on their energy content. Sirovich (1987) presented the snapshot POD, which relaxes the computational difficulties of the classical orthogonal decomposition. POD has been used to describe coherent structures for different flows, such as axisymmetric mixing layer (Glauser and George, 1987), channel flow (Moin and Moser, 1989), atmospheric boundary layer (Shah and Bou-Zeid, 2014), wake behind disk (Tutkun et al., 2008), and a wind turbine wake flow 
(Andersen et al., 2013; Bastine et al., 2014; VerHulst and Meneveau, 2014; Hamilton et al., 2015b; Ali et al., 2016b, 2017b).

The flow field, taken as the fluctuating velocity after subtracting time-average mean velocity from instantaneous velocity, can be represented as $u=u\left(\boldsymbol{x}, t^{n}\right)$, where $\boldsymbol{x}$ and $t^{n}$ refer to the spatial coordinates and time at sample $n$, respectively. A set of the orthonormal basis functions, $\phi$, can be presented as

$\phi=\sum_{n=1}^{N} A\left(t^{n}\right) u\left(\boldsymbol{x}, t^{n}\right)$,

where $N$ is the number of snapshots. The largest projection can be determined using the two-point correlation tensor and Fredholm integral equation

$\int_{\Omega} \frac{1}{N} \sum_{n=1}^{N} u\left(\boldsymbol{x}, t^{n}\right) u^{T}\left(\boldsymbol{x}^{\prime}, t^{n}\right) \phi\left(\boldsymbol{x}^{\prime}\right) \mathrm{d} \boldsymbol{x}=\lambda \phi(\boldsymbol{x})$,

where the left-hand side of the equation presents a spatial correlation between two points $\boldsymbol{x}$ and $\boldsymbol{x}^{\prime}, T$ signifies the transpose of a matrix, $\Omega$ is the physical domain, and $\lambda$ represents the eigenvalues. To acquire the optimal basis functions, the problem is reduced to an eigenvalue decomposition denoted as $[C][G]=\lambda[G]$, where $C, G$, and $\lambda$ are the correlation tensor, basis of eigenvectors, and eigenvalues, respectively. The matrix [G] is related to the time coefficient as $[G]=\left[A\left(t^{1}\right), A\left(t^{2}\right), \cdots, A\left(t^{N}\right)\right]^{T}$. The POD eigenvectors illustrate the spatial structure of the turbulent flow and the eigenvalues measure the energy associated with corresponding eigenvectors. The summation of the eigenvalues presents the total turbulent kinetic energy $(E)$ in the flow domain. The cumulative kinetic energy fraction $\eta$ and the normalized energy content of each mode $\xi$ can be represented as $\eta_{n}=\sum_{j=1}^{n} \lambda_{n} / \sum_{j=1}^{N} \lambda_{n}$ and $\xi_{n}=\lambda_{n} / \sum_{j=1}^{N} \lambda_{n}$. POD is particularly useful in rebuilding the Reynolds shear stress using a limited set $\left(N_{\mathrm{lm}}\right)$ of eigenfunctions as

$\left\langle u_{i} u_{j}\right\rangle=\sum_{n=1}^{N_{\operatorname{lm}}} \lambda_{n} \phi_{i}^{n} \phi_{j}^{n}$

\subsection{Reynolds stress anisotropy}

Following the development presented by Rotta (1951), the Reynolds stress anisotropy tensor is written $a_{i j}=\overline{u_{i} u_{j}}-$ $\frac{2}{3} k \delta_{i j}$, where $\delta_{i j}$ is the Kronecker delta and $k$ represents the turbulence kinetic energy and is defined by $k=0.5 \sum_{i=1}^{3}\left\langle u_{i} u_{i}\right\rangle$. The deviatoric tensor is then $b_{i j}=$ $\overline{u_{i} u_{j}} / \overline{u_{k} u_{k}}-\frac{1}{3} \delta_{i j}$, of which the second and third scalar invariants are determined as $6 \eta^{2}=b_{i j} b_{j i}$ and $6 \xi^{3}=b_{i j} b_{j k} b_{k i}$, respectively (Pope, 2000; Lumley and Newman, 1977). The second invariant, $\eta$, measures the degree of the anisotropy
Table 1. Summary of the special turbulence cases described by the barycentric map.

\begin{tabular}{ll}
\hline Cases & Eigenvalues \\
\hline Three components & $\lambda_{1}=\lambda_{2}=\lambda_{3}=0$ \\
Two components & $\lambda_{1}=\lambda_{2}=\frac{1}{6}, \lambda_{3}=-\frac{1}{3}$ \\
One component & $\lambda_{1}=\frac{2}{3}, \lambda_{2}=\lambda_{3}=-\frac{1}{3}$ \\
\hline
\end{tabular}

and the third invariant, $\xi$, specifies the state of turbulence. Alternatively, the eigenvalue decomposition of the normalized Reynolds stress anisotropy tensor $b_{i j}$ can be used to derive the second and third invariants as $\eta^{2}=\frac{1}{3}\left(\lambda_{1}^{2}+\lambda_{1} \lambda_{2}+\lambda_{2}^{2}\right)$ and $\xi^{3}=-\frac{1}{2} \lambda_{1} \lambda_{2}\left(\lambda_{1}+\lambda_{2}\right)$. In an attempt to further facilitate the study of turbulence anisotropy, Banerjee et al. (2007) presented a linearized anisotropy tensor invariant, termed barycentric map (BM), as

$$
\begin{aligned}
\hat{b}_{i j} & =C_{1 c}\left(\begin{array}{ccc}
2 / 3 & 0 & 0 \\
0 & -1 / 3 & 0 \\
0 & 0 & -1 / 3
\end{array}\right) \\
& +C_{2 c}\left(\begin{array}{ccc}
1 / 6 & 0 & 0 \\
0 & 1 / 6 & 0 \\
0 & 0 & -1 / 3
\end{array}\right)+C_{3 c}\left(\begin{array}{lll}
0 & 0 & 0 \\
0 & 0 & 0 \\
0 & 0 & 0
\end{array}\right),
\end{aligned}
$$

where $C_{1 c}, C_{2 c}$, and $C_{3 c}$ are the coefficients that represent the boundaries of the barycentric map. The BM coefficients are determined as $C_{1 c}=\lambda_{1}-\lambda_{2}, C_{2 c}=2\left(\lambda_{2}-\lambda_{3}\right)$, and $C_{3 c}=3 \lambda_{3}+1$. The basis matrices in Eq. (4) represent the vertices of an equilateral triangle with coordinates $\left(x_{1 c}, y_{1 c}\right)$, $\left(x_{2 c}, y_{2 c}\right)$, and $\left(x_{3 c}, y_{3 c}\right)$. Table 1 presents the states of turbulence that correspond to each vertex of the BM, describing either isotropic (three-component), one-component, or twocomponent turbulence. As a result, any realizable turbulence state can be represented as follows:

$x_{\text {new }}=C_{1 c} x_{1 c}+C_{2 c} x_{2 c}+C_{3 c} x_{3 c}$,
$y_{\text {new }}=C_{1 c} y_{1 c}+C_{2 c} y_{2 c}+C_{3 c} y_{3 c}$.

Emory and Iaccarino (2014) also introduced a color-mapbased visualization technique that aids in interpreting the spatial distribution of the normalized anisotropy tensor. In this case, they attributed to each vertex of the barycentric map an RGB (red-green-blue) color scale; see Fig. 1 for more details. This color map technique combines the coefficients $C_{1 c}, C_{2 c}$, and $C_{3 c}$ to generate an RGB map such that

$\left[\begin{array}{l}R \\ G \\ B\end{array}\right]=C_{1 c}^{*}\left[\begin{array}{l}1 \\ 0 \\ 0\end{array}\right]+C_{2 c}^{*}\left[\begin{array}{l}0 \\ 1 \\ 0\end{array}\right]+C_{3 c}^{*}\left[\begin{array}{l}0 \\ 0 \\ 1\end{array}\right]$,

where $C_{i c}^{*}$ are the modified coefficients that can be determined as $C_{i c}^{*}=\left(C_{i c}+0.65\right)^{5}$. The coefficient with a value 


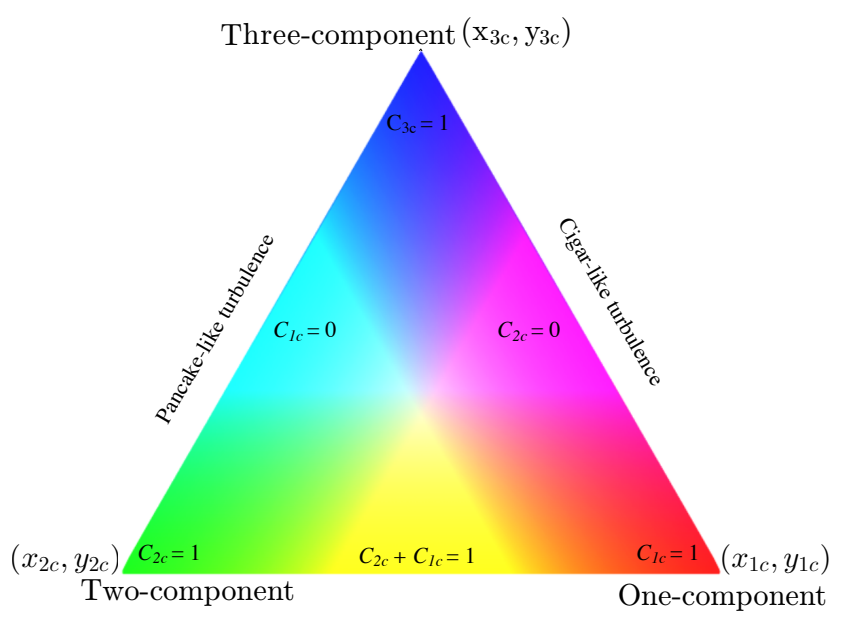

Figure 1. Schematic representation of the barycentric map (BM) with color.

of 0.65 and 5 is applied as it provides the optimal visualization; other coefficients are tested with less success in terms of marking differences. As a result, one-component turbulence is associated with the red color, two-component turbulence with green, and three-component (isotropic turbulence) with blue; see Fig. 1. The anisotropy has been examined in different types of flow, including pipe and duct flows (Antonia et al., 1991; Krogstad and Torbergsen, 2000), the atmospheric boundary layer (Klipp, 2010, 2012), and the wake of a wind turbine (Gómez-Elvira et al., 2005; Hamilton and Cal, 2015; Ali et al., 2017a, 2018). Here the anisotropy stress tensor is employed to quantify the effect of the spacing on the turbulence states.

\section{Experimental design}

A $4 \times 3$ array of wind turbines was placed in the closed-circuit wind tunnel at Portland State University to study the effects due to variation in streamwise and spanwise spacing in a wind turbine array. The dimensions of the wind tunnel test section were $5 \mathrm{~m}$ (long), $1.2 \mathrm{~m}$ (wide), and $0.8 \mathrm{~m}$ (high). The blockage ratio comparing the frontal area of the model wind turbines to the cross-sectional area of the test section was less than $5 \%$. The entrance of the test section was conditioned by the passive grid, which consists of seven horizontal and six vertical rods, to introduce large-scale turbulence. Nine vertical acrylic strakes, located $0.25 \mathrm{~m}$ downstream of the passive grid and $2.15 \mathrm{~m}$ upstream of the first row of the wind turbine, were used to modify the inflow. The thickness of the strakes was $0.0125 \mathrm{~m}$ and they are spaced every $0.136 \mathrm{~m}$ across the test section. Surface roughness was introduced to the wall as a series of chains with a diameter of $0.0075 \mathrm{~m}$, spaced $0.11 \mathrm{~m}$ apart. Figure 2 shows the schematic of the experimental setup.
Table 2. Streamwise and spanwise spacing of the experimental tests.

\begin{tabular}{lccc}
\hline Cases & $S_{x}$ & $S_{z}$ & Occupied area \\
\hline $\mathrm{C}_{6 \times 3}$ & $6 D$ & $3 D$ & $18 D^{2}$ \\
$\mathrm{C}_{3 \times 3}$ & $3 D$ & $3 D$ & $9 D^{2}$ \\
$\mathrm{C}_{3 \times 1.5}$ & $3 D$ & $1.5 D$ & $4.5 D^{2}$ \\
$\mathrm{C}_{6 \times 1.5}$ & $6 D$ & $1.5 D$ & $9 D^{2}$ \\
\hline
\end{tabular}

Sheet steel $0.0005 \mathrm{~m}$ thick was used to construct the threebladed wind turbine rotors. The diameter of the rotor was $D=0.12 \mathrm{~m}$, equal to the height of the turbine tower. The scaled turbine models were manufactured in-house. Based on full-scale turbines with a $100 \mathrm{~m}$ rotor diameter and a $100 \mathrm{~m}$ hub height, the models were built on a $1: 830$ scale. In this study, the Reynolds number in the entrance row turbines was approximately the same order of magnitude of the independent range detailed in Chamorro et al. (2012). The rotor blades were steel sheets laser cut to shape and were $0.0005 \mathrm{~m}$ thick. The blades were shaped using a die press. The die press was designed in-house to produce a $15^{\circ}$ pitch from the plane of the rotor and a $10^{\circ}$ twist at the tip. Figure 3 presents the schematic of the wind turbine model. The wind turbine model design used is that presented in Cal et al. (2010), Kang and Meneveau (2010), and Hamilton et al. (2015a). Operating conditions for the wind turbines were also scaled, namely the power coefficient, $C_{\mathrm{p}}$, and tip-speed ratio, $\lambda$, which were detailed in Hamilton et al. (2015a). The streamwise integral length scale is approximately $0.13 \mathrm{~m}$, which was the same order of magnitude as the turbine rotor and representative of conditions seen by full-scale turbines in atmospheric flows. A DC electrical motor of $0.0013 \mathrm{~m}$ diameter and $0.0312 \mathrm{~m}$ long formed the nacelle of the turbine and was aligned with the flow direction. A torque-sensing system was connected to the DC motor shaft following the design outlined in Kang and Meneveau (2010). The torque sensor consists of a strain gauge, Wheatstone bridge, and data acquisition with measuring software to collect the data.

The flow field was sampled in four configurations of a model-scale wind turbine array, classified as $C_{S_{x} \times S_{z}}$, shown in Table 2. Permutations of the streamwise spacing $\left(S_{x}\right)$ of 6 and $3 D$ and spanwise spacing $\left(S_{z}\right)$ of 3 and $1.5 D$ are examined. Stereoscopic particle image velocimetry (SPIV) was used to measure streamwise, wall-normal, and spanwise instantaneous velocity upstream and downstream of the wind turbine at the center line of the fourth row as shown in Fig. 4. At each measurement location, 2000 images were taken to ensure convergence of second-order statistics. The nominal sampling rate of the SPIV system is fixed at $5 \mathrm{~Hz}$. The SPIV system consists of a Nd: YAG $(532 \mathrm{~nm}, 1200 \mathrm{~mJ}$, 4 ns duration) double-pulsed laser and four $4 \mathrm{Mpx}$ Imager ProX CCD cameras arranged in pairs upstream and downstream of the wind turbine. Neutrally buoyant fluid particles 


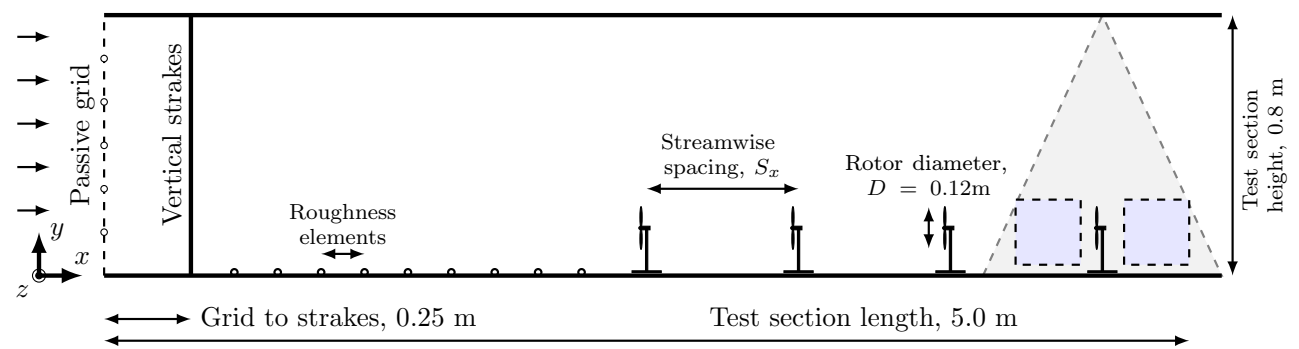

Figure 2. Experimental setup. Dashed gray lines indicate the placement of the laser sheet relative to the model wind turbine array. Filled gray boxes indicate measurement locations discussed below.

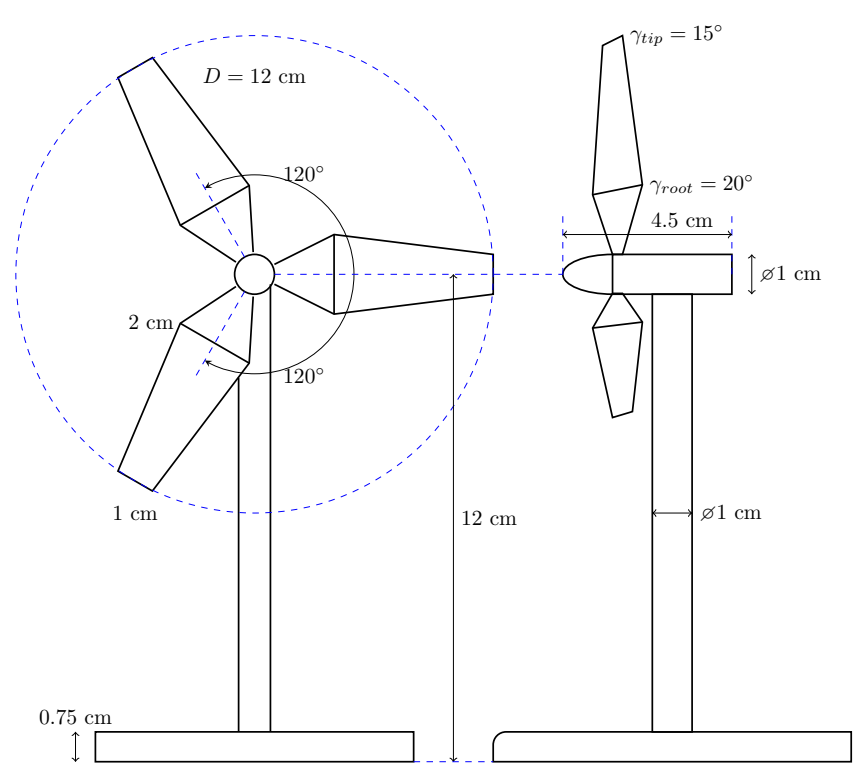

Figure 3. Schematic representation of the wind turbine model.

of diethyl hexyl sebacate were introduced to the flow and allowed to mix. Consistent seeding density was maintained in order to mitigate measurement errors. The laser sheet was approximately $0.001 \mathrm{~m}$ thick with a divergence angle of less than $5 \mathrm{mrad}$. Each measurement window was $0.2 \mathrm{~m} \times 0.2 \mathrm{~m}$ aligned with the center of each turbine, parallel to the bulk flow. A multi-pass fast Fourier transformation was used to process the raw data into vector fields. Erroneous measurement of the vector fields was replaced using Gaussian interpolation of neighboring vectors. Based on the variability estimator (George, 2013), the error of the SPIV measurements was on the order of $3 \%$ with the greatest uncertainty pertaining to the out-of-plane (spanwise) component.

\section{Results}

\subsection{Statistical analysis}

Characterization of the wind turbine wake flow is presented by the streamwise mean velocity and Reynolds shear stress, with the aim to understand the influence of turbine-to-turbine spacing. Figure 5 presents the streamwise normalized mean velocity, $U / U_{\infty}$, upstream and downstream of each wind turbine for the cases $\mathrm{C}_{6 \times 3}, \mathrm{C}_{3 \times 3}, \mathrm{C}_{3 \times 1.5}$, and $\mathrm{C}_{6 \times 1.5}$. The inflow mean velocity at the hub height $U_{\infty}=5.5 \mathrm{~m} \mathrm{~s}^{-1}$ is used in the normalization. For each turbine, the flow upstream and downstream is shown by the contour plots on the left and right, respectively. In the upstream region, case $\mathrm{C}_{6 \times 3}$ exhibits the largest streamwise mean velocities due to greater recovery of the flow upstream of the turbine. Although the streamwise spacing of case $\mathrm{C}_{6 \times 1.5}$ is the same as case $\mathrm{C}_{6 \times 3}$, the former shows reduced hub height velocity. The normalized mean velocity is about 0.567 compared with 0.66 in case $\mathrm{C}_{6 \times 3}$, showing the influence of the spanwise spacing on wake evolution and flow recovery. Variations between case $\mathrm{C}_{3 \times 3}$ and $\mathrm{C}_{3 \times 1.5}$ are small. Downstream of the turbine, the four cases show differences outside of the rotor area, where case $\mathrm{C}_{6 \times 3}$ shows the greatest velocities by approximately $20 \%$. Case $\mathrm{C}_{3 \times 3}$ also shows higher velocities below the bottom tip compared with cases $\mathrm{C}_{3 \times 1.5}$ and $\mathrm{C}_{6 \times 1.5}$. The normalized mean streamwise velocity and the turbulence intensity in Nilsson et al. (2015) showed similar compound wakes from the upstream and downstream turbines and confirmed the current result of cases $\mathrm{C}_{3 \times 3}$ and $\mathrm{C}_{3 \times 1.5}$. In that study, there was one location with an absent turbine and the flow was given extra space for recovery. The recovered wake flow in Nilsson et al. (2015) is similar to the present cases $\mathrm{C}_{6 \times 3}$ and $\mathrm{C}_{6 \times 1.5}$.

Figure 6 compares the in-plane normalized Reynolds shear stress $-\overline{u v} / U_{\infty}^{2}$ for all test cases. The fluctuating velocities in the streamwise and wall-normal directions are denoted as $u$ and $v$, respectively. In the upstream window, cases $\mathrm{C}_{3 \times 3}$ and $\mathrm{C}_{3 \times 1.5}$ display higher values of the stress compared with $\mathrm{C}_{6 \times 3}$ and $\mathrm{C}_{6 \times 1.5}$ cases. Although the spanwise spacing of case $\mathrm{C}_{3 \times 1.5}$ is half of case $\mathrm{C}_{3 \times 3}$, no relevant differences are apparent. In the downstream window, comparison indicates that reducing streamwise spacing increases the Reynolds shear stress. The average value of the shear stress in the wake is $16 \%$ greater for $\mathrm{C}_{3 \times 3}$ than for $\mathrm{C}_{6 \times 3}$. A similar effect is observed in case $\mathrm{C}_{3 \times 1.5}$, in which the average value of the stress is $2 \%$ greater than that of $\mathrm{C}_{6 \times 1.5}$. The effect of spanwise spacing is more pronounced when the streamwise 


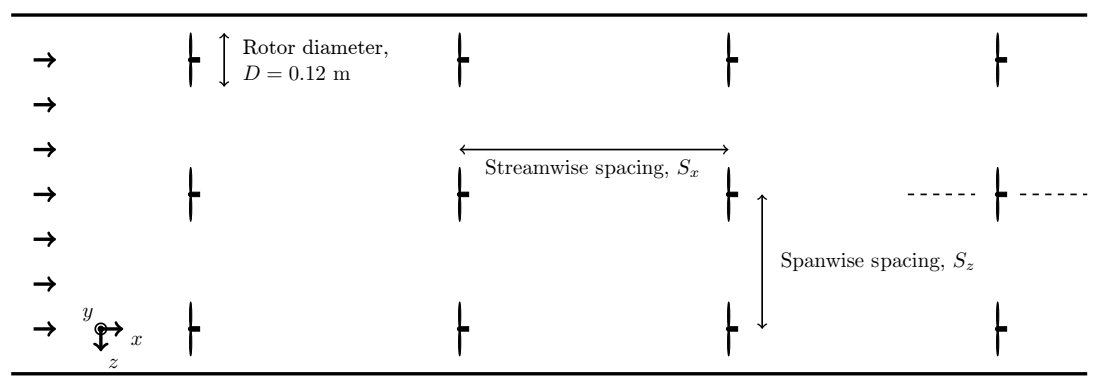

Figure 4. Top view of the 4 by 3 wind turbine array. The dashed lines at the last row centerline turbine represent the measurement locations.
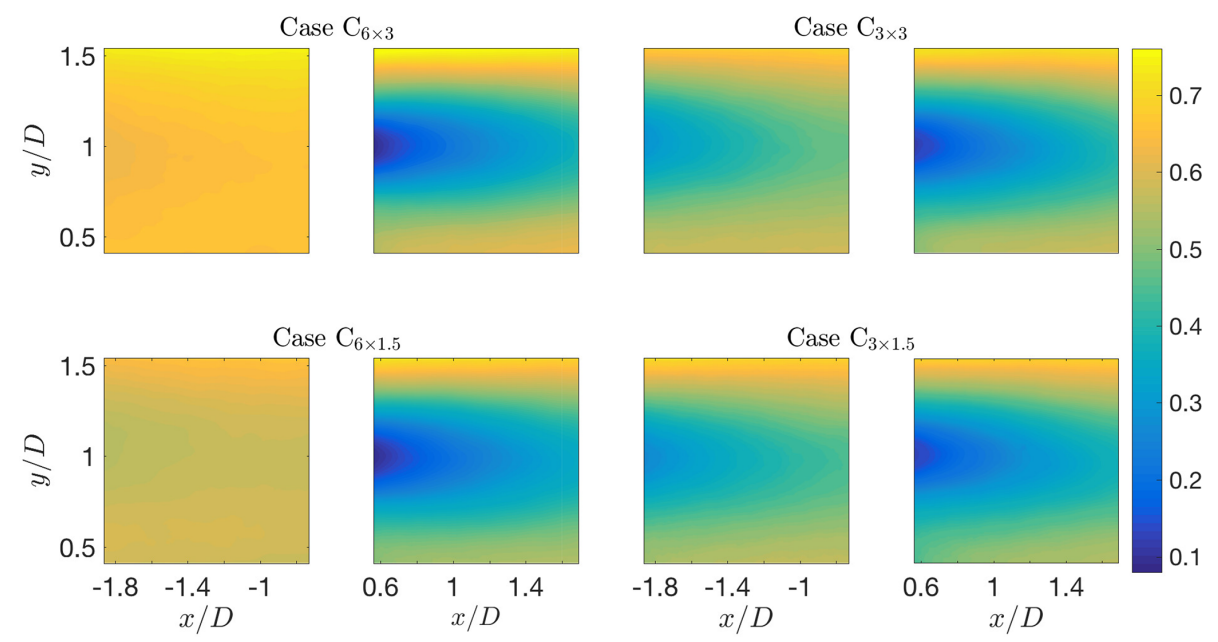

Figure 5. Normalized streamwise velocity, $U / U_{\infty}$, upstream and downstream of cases $\mathrm{C}_{6 \times 3}, \mathrm{C}_{3 \times 3}, \mathrm{C}_{3 \times 1.5}$, and $\mathrm{C}_{6 \times 1.5}$.

spacing is $3 D$; the average shear stress is approximately $20 \%$ greater in $\mathrm{C}_{3 \times 1.5}$ than in $\mathrm{C}_{3 \times 3}$.

\subsection{Averaged profiles}

Spatial averaging of the flow statistics is undertaken by moving the upstream domain of each case beyond its corresponding downstream domain and performing streamwise averaging, following the procedure in Cal et al. (2010). Through spatial averaging, it is possible to compare key data from different cases taking into account the different streamwise spacings. Streamwise averaging is denoted by $\langle\cdot\rangle_{x}$. Figure $7 \mathrm{a}$ shows profiles of streamwise-averaged mean velocity for all four cases. Cases $\mathrm{C}_{6 \times 3}$ and $\mathrm{C}_{3 \times 1.5}$ show the largest and smallest velocity deficits, respectively. At hub height, the velocity of case $\mathrm{C}_{6 \times 3}$ is approximately $2.25 \mathrm{~m} \mathrm{~s}^{-1}$ whereas case $\mathrm{C}_{3 \times 1.5}$ shows a velocity of approximately $1.6 \mathrm{~m} \mathrm{~s}^{-1}$. Comparing to $\mathrm{C}_{6 \times 3}$, the change seen in the spatially averaged velocity is greater in $\mathrm{C}_{3 \times 3}$ than in $\mathrm{C}_{6 \times 1.5}$, confirming that the impact of reducing streamwise spacing is greater than changing the spanwise spacing. Interestingly, when the spanwise spacing is fixed to $S_{z}=1.5 \mathrm{D}$, changing the streamwise spacing has an effect smaller than expected. Constraining the wake suppresses development of the mean velocity in the streamwise and spanwise directions.

Figure $7 \mathrm{~b}$ contains the streamwise-averaged Reynolds shear stress $\left\langle-\overline{u v} / U_{\infty}^{2}\right\rangle_{x}$ for cases $\mathrm{C}_{6 \times 3}$ through $\mathrm{C}_{6 \times 1.5}$. Slightly decreased values of $\left\langle-\overline{u v} / U_{\infty}^{2}\right\rangle_{x}$ are seen in case $\mathrm{C}_{6 \times 1.5}$, where the spanwise spacing is reduced, especially below the turbine hub height $y / D=1$. Reducing spanwise spacing shows a more pronounced effect when the streamwise spacing is $S_{x}=3 \mathrm{D}$. The streamwise spacing plays a larger role than the spanwise spacing, i.e., the maximum differences between the Reynolds shear stress profiles are detected between cases $\mathrm{C}_{6 \times 3}$ and $\mathrm{C}_{3 \times 3}$. Interestingly, the largest difference between the spatially averaged Reynolds shear stress is found between cases $\mathrm{C}_{6 \times 3}$ and $\mathrm{C}_{3 \times 3}$, located at $y / D \approx 0.7$ and $y / D \approx 1.4$. Furthermore, the four cases have approximately zero Reynolds shear stress at the inflection point located at hub height. In addition, case $\mathrm{C}_{3 \times 3}$ displays the maximum Reynolds stress and case $\mathrm{C}_{6 \times 1.5}$ presents the minimum stress.

\subsection{Proper orthogonal decomposition}

Eigenvalues produced in the POD express the integrated turbulence kinetic energy associated with basis function de- 

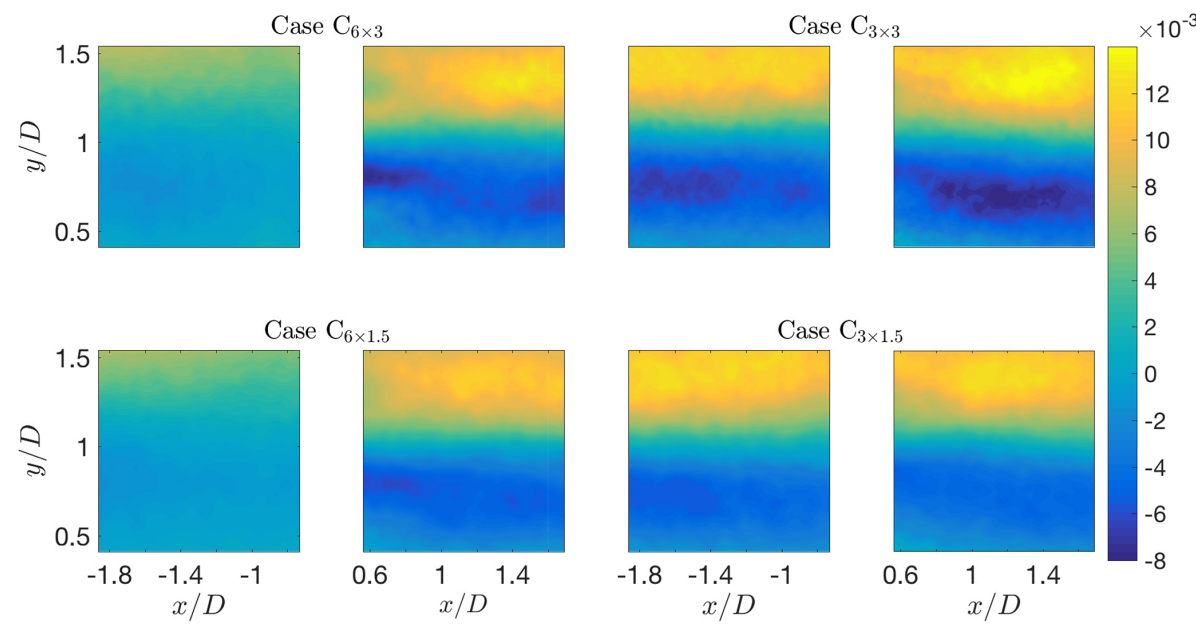

Figure 6. Normalized Reynolds shear stress, $-\overline{u v} / U_{\infty}^{2}$, upstream and downstream of each measurement case.
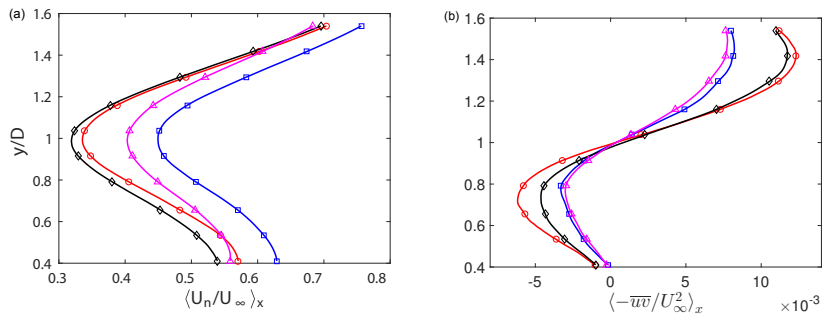

Figure 7. Streamwise-averaged profiles of streamwise velocity and Reynolds shear stress for four different cases: $\mathrm{C}_{6 \times 3}$ (blue $\square$ ), $\mathrm{C}_{3 \times 3}$ (red $\bigcirc), \mathrm{C}_{3 \times 1.5}($ black $\diamond)$, and $\mathrm{C}_{6 \times 1.5}($ pink $\triangle$ ).

scribing the flow. The normalized cumulative energy fractions $\eta_{n}$ for upstream and downstream measurement windows are presented in Fig. 8a and b, respectively. Inset figures exhibit the normalized energy content per mode, $\xi_{n}$. Upstream of the turbine, cases $\mathrm{C}_{6 \times 3}$ and $\mathrm{C}_{6 \times 1.5}$ converge more toward $\eta_{n}$ than cases $\mathrm{C}_{3 \times 3}$ and $\mathrm{C}_{3 \times 1.5}$, respectively. These results are attributed to the reduction in the streamwise spacing. The convergence of case $\mathrm{C}_{3 \times 3}$ is approximately coincident with that of case $C_{3 \times 1.5}$. In the downstream measurement window, case $\mathrm{C}_{6 \times 1.5}$ converges faster than the other cases, followed by $\mathrm{C}_{6 \times 3}, \mathrm{C}_{3 \times 3}$, and $\mathrm{C}_{3 \times 1.5}$. The comparison between the upstream and downstream windows reveals that energy accumulates in fewer modes upstream in every test case, e.g., case $\mathrm{C}_{6 \times 3}$ requires 14 modes to obtain $50 \%$ of the total kinetic energy in the upstream window, whereas 26 modes are required to obtain the same percentage of energy downstream of the turbine. Cases $\mathrm{C}_{6 \times 1.5}$ and $\mathrm{C}_{3 \times 1.5}$ show the respective maximum and minimum variations in $\lambda_{1}$ between upstream and downstream measurements. This observation can be attributed to the structure of the upstream flow of case $\mathrm{C}_{6 \times 1.5}$, which is more recovered compared to the downstream flow, where the turbulence is high in energy content and more complex. However, the upstream and downstream
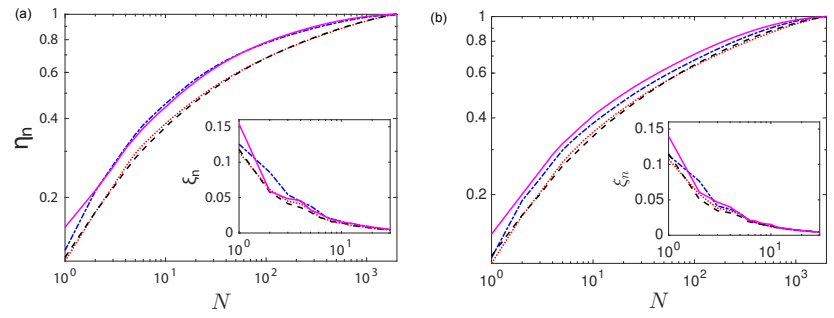

Figure 8. Energy content of the POD modes for four different cases: $\mathrm{C}_{6 \times 3}$ (blue), $\mathrm{C}_{3 \times 3}$ (red), $\mathrm{C}_{3 \times 1.5}$ (black), and $\mathrm{C}_{6 \times 1.5}$ (pink).

windows of case $\mathrm{C}_{3 \times 1.5}$ are more similar in terms of turbulence and organization. From mode 2 through 10, the starkest difference between the upstream and downstream is found in case $\mathrm{C}_{6 \times 3}$. Increasing the characteristic area per turbine provides room for the flow to become more homogeneous in the upstream window and exhibit the most significant momentum deficit in the wake, accounting for the differences seen in $\eta_{n}$ upstream and downstream.

The streamwise component of the selected POD modes is shown for all cases in Figs. 9 through 11. These modes are selected because they provide a range of large and intermediate scales and highlight the discrepancies among the cases. Figure 9 presents the first POD mode upstream and downstream of the considered cases. The four cases show small gradients in the streamwise direction compared to a large gradient in the wall-normal direction. Although the four cases show a divergence between the eigenvalues of the first mode, the eigenfunctions demonstrate very similar structure. For case $\mathrm{C}_{6 \times 3}$, the energy of the first POD mode decreases by $1.25 \%$, comparing the upstream eigenvalue to the downstream one; see Fig. 8. Smaller variations of 0.68 and $0.32 \%$ are observed in cases $\mathrm{C}_{3 \times 3}$ and $\mathrm{C}_{3 \times 1.5}$, respectively. Consequently, the structures upstream and downstream of these cases are approximately equivalent. The upstream measure- 

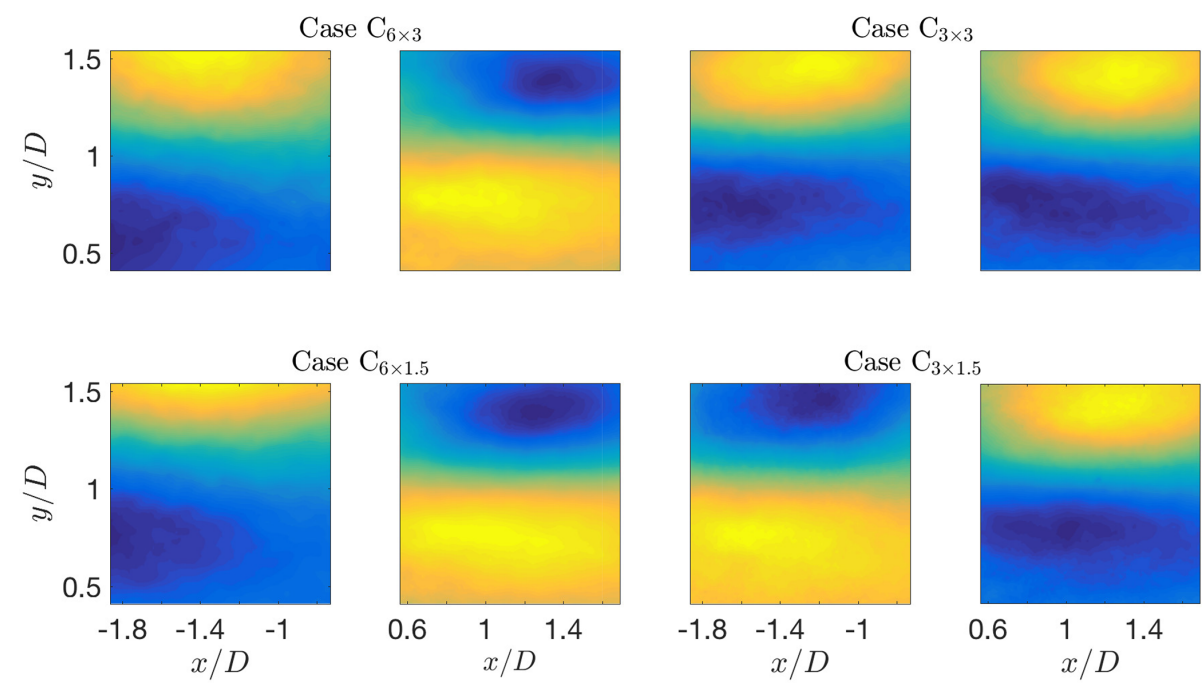

Figure 9. The first mode upstream and downstream of the each case.
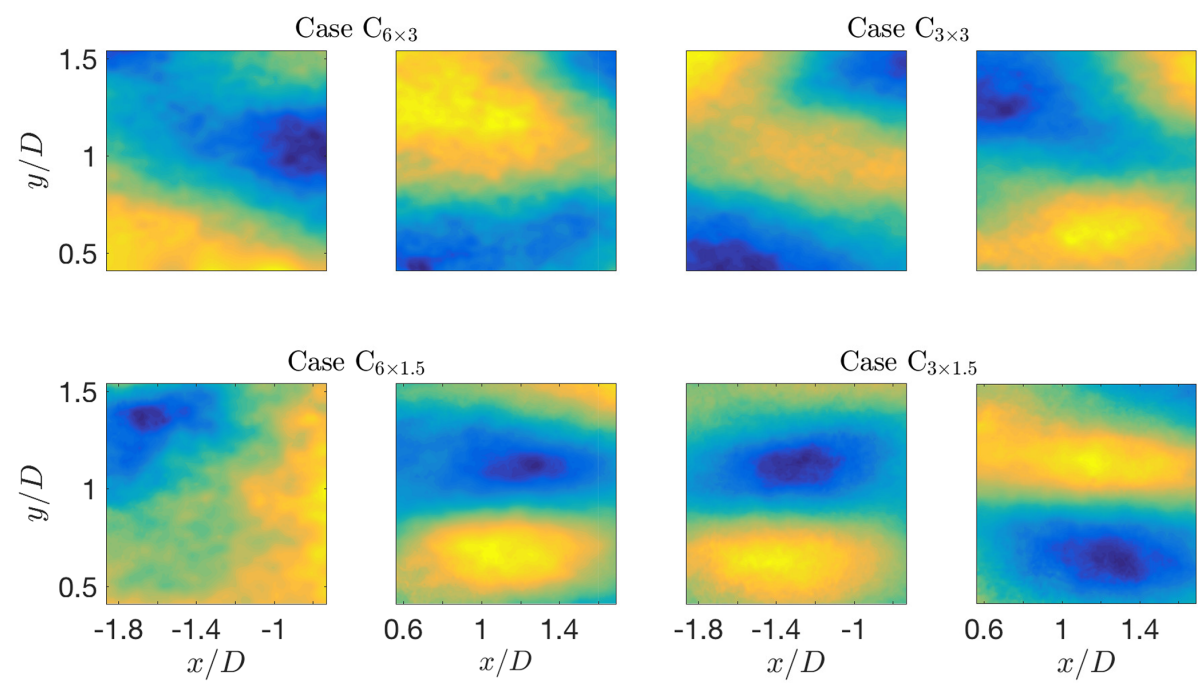

Figure 10. The fifth mode upstream and downstream of the each case.

ment domain of cases $\mathrm{C}_{6 \times 3}$ and $\mathrm{C}_{6 \times 1.5}$ is representative of the recovering part of the flow, in contrast to the downstream part that presents the wake region. This difference in the physical space has an impact on the low-number POD modes that show a discrepancy in the coherent structures between the upstream and downstream windows. In the $\mathrm{C}_{3 \times 3}$ arrangement, upstream and downstream regions exhibit similar behavior, thus pointing to the resemblance in the structure. Alike observations can be extracted from case $\mathrm{C}_{3 \times 1.5}$. Of note, a difference in sign of the eigenvectors is present, which is one of the POD properties.

Figure 10 presents the fifth POD mode of the four cases that show a combination of POD and Fourier (homogenous) modes in the streamwise direction. Although the fifth mode of the four cases contains $\approx 74 \%$ less energy than the first mode, large scales are still pronounced. Smaller features also appear in the upstream and the downstream windows. The upstream window of cases $\mathrm{C}_{6 \times 3}, \mathrm{C}_{3 \times 3}$, and $\mathrm{C}_{3 \times 1.5}$ is shifted horizontally in the downstream window. The upstream and downstream windows of case $\mathrm{C}_{3 \times 1.5}$ look like the first mode, reduced in size, as is observed in the downstream window of the case $\mathrm{C}_{6 \times 1.5}$.

Figure 11 presents the 20th POD mode, in which small structures become noticeable in both upstream and downstream windows. The upstream measurement window of cases $\mathrm{C}_{6 \times 3}$ and $\mathrm{C}_{6 \times 1.5}$ shows larger-scale structures compared to the other two cases. Although, after mode 10, there is no significant difference in the energy content from case to case, the structure of the modes shows a significant discrepancy between the cases, confirming that the intermediate 

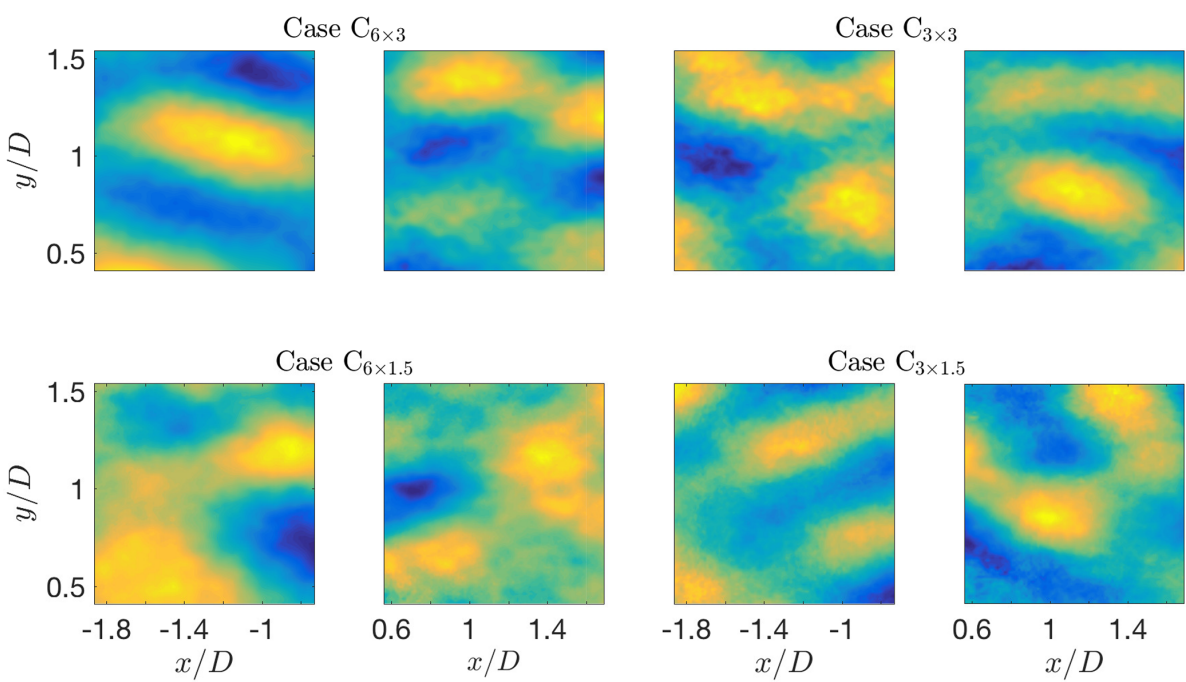

Figure 11. The 20th mode upstream and downstream of the each case.

modes are associated with the inflow characterizations. Thus, the intermediate modes are responsible for carrying the significant part of flow dynamic and cooperative behavior in the energy cascade. Therefore, any low-order models should include these intermediate modes in order to improve the behavior dramatically and capture the dynamic of the full system.

\subsection{Reconstruction of averaged profile}

Combining the POD modes with the corresponding time coefficient gives these modes physical interpretation and shows the contribution of the modes to the overall flow behavior. A reduced degree of the turbulence kinetic energy is considered using only a few modes to reconstruct the streamwiseaveraged profiles of Reynolds shear stress. Reconstructions are made using either a single mode or the first 5, 10, 25, or 50 modes to represent the stress, shown in Fig. 12. Inset figures present the Reynolds shear stress construction using modes 5-10, 5-25, and 5-50, excluding the first four modes to isolate contributions from intermediate modes. The black lines are the streamwise-averaged stresses from the full data in Fig. 7b. Using an equal number of modes, case $\mathrm{C}_{6 \times 1.5}$ rebuilds the profiles of the Reynolds shear stress faster than the other cases. Case $\mathrm{C}_{6 \times 3}$ also shows a faster reconstruction and dissimilarity to case $\mathrm{C}_{6 \times 1.5}$, mainly arising from the profile of the first mode (red line). Cases $\mathrm{C}_{3 \times 3}$ and $\mathrm{C}_{3 \times 1.5}$ show approximately the same trends in reconstruction profiles. Below hub height, the four cases show the same trend of the first-mode profiles, where the contribution in the reconstruction profiles is zero. The maximum difference between the successive reconstruction profiles occurs between the first mode and the first five modes. Cases $\mathrm{C}_{6 \times 3}, \mathrm{C}_{3 \times 3}$, and $\mathrm{C}_{3 \times 1.5}$ show moderate variation between the profiles of the reconstructed stress resulting from the first five and first
10 modes (red and green lines, respectively). After mode 10, contributions by each additional mode are quite small, shown by pink and gray lines.

The maximum difference between the full data and the reconstructed profiles is located at $y / D \approx 0.75$ and $y / D \approx 1.4$, where the extrema in $\langle-\overline{u v}\rangle_{x}$ are located. Generally, faster reconstruction implies that the flow possesses coherent structures with a greater portion of the total kinetic energy. Consequently, the flow characterized with greater coherence in cases $\mathrm{C}_{6 \times 3}$ and $\mathrm{C}_{6 \times 1.5}$. In cases $\mathrm{C}_{3 \times 3}$ and $\mathrm{C}_{3 \times 1.5}$, fewer energetic features arise from the reduced spacing effect, which leads to a reduction of the mean velocities within the canopy and an increase in lateral wake interactions. These interactions, which become larger as a result of the accumulated wakes, expand downstream of the rotor. Thus, the streamwise spacing allows for the flow to recover and therefore produce larger, more coherent structures within the domain, which in comparison eclipses variations produced by the spanwise spacing. Also, the large spacing offers a larger frontal area to the wind coming from above the lateral sides.

To quantify the contribution of the moderately scaled structures, the Reynolds shear stress is reconstructed using the intermediate modes. As can be shown in the insets of Fig. 12, the full data profile (black line) is compared with profiles reconstructed from modes 5-10 (red line), 5-25 (blue line), and 5-50 (green lines). The intermediate modes in each case approximately take the form of the full data profiles below the hub height, although the magnitudes of the reconstructions are smaller than those of the full data statistics. Reconstruction Reynolds shear stress in cases $\mathrm{C}_{6 \times 3}$ and $\mathrm{C}_{3 \times 1.5}$ shows minute variations between the reconstructed profiles and is essentially a vertical line above the hub height. This trend is opposite that shown by the profile of the first mode alone, indicating that the most energetic modes selectively reconstruct turbulence above hub height. Cases $C_{3 \times 3}$ 

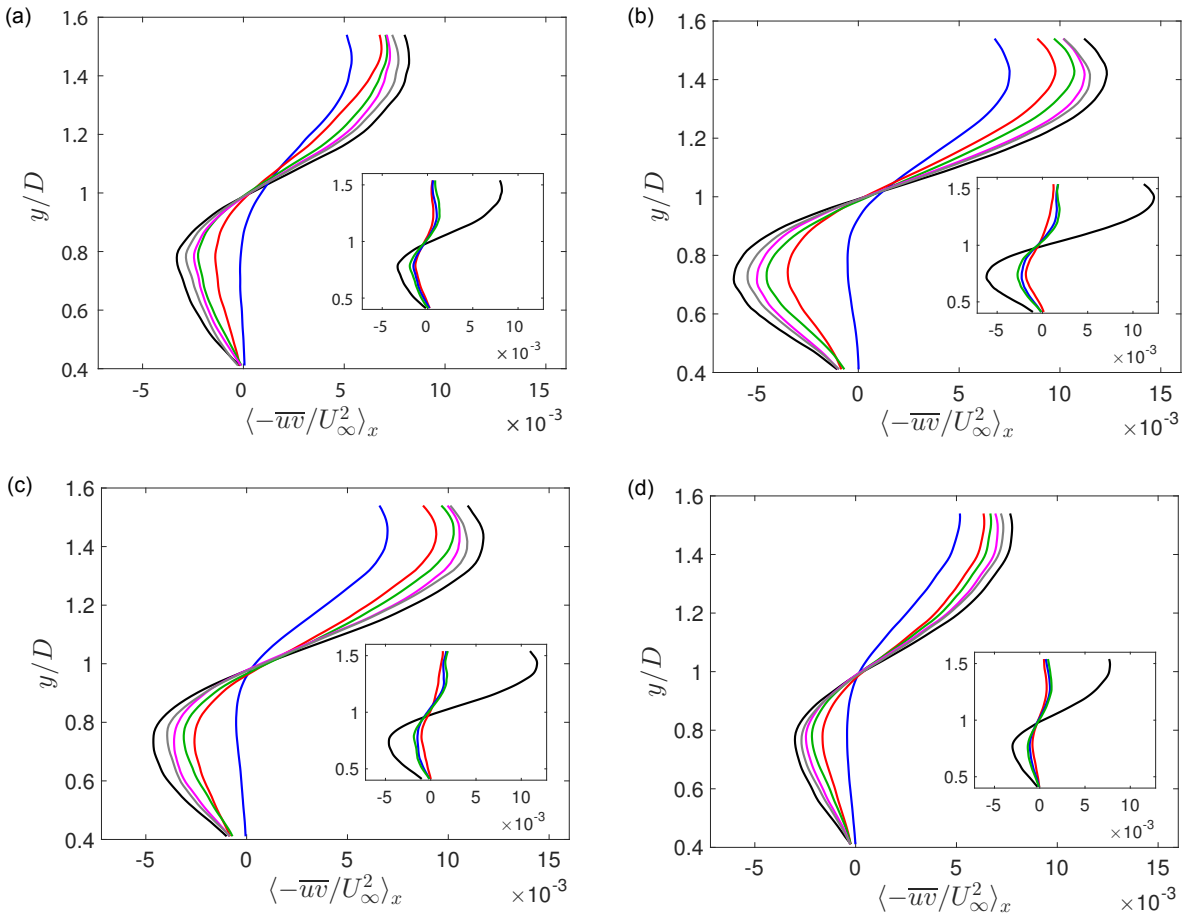

Figure 12. Reconstruction Reynolds shear stress using the first mode (blue), first five modes (red), first 10 modes (green), first 25 modes (pink), and first 50 modes (gray). Full data statistics (black). The insets show the reconstruction using modes 5-10 (red), 5-25 (blue), and 5-50 (green).
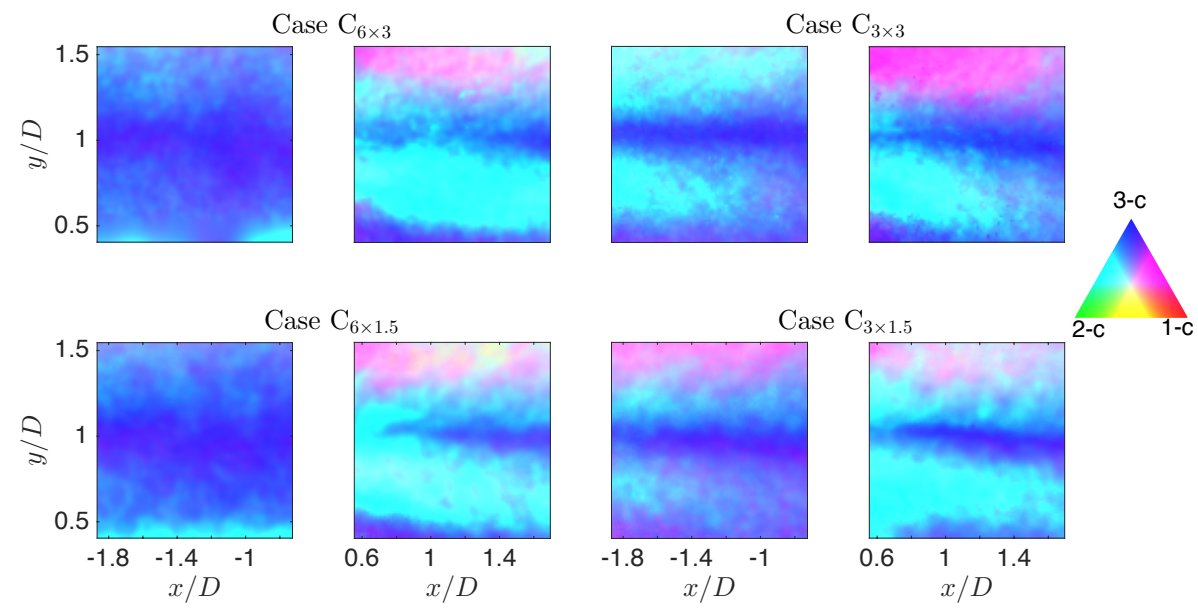

Figure 13. Barycentric map for upstream and downstream of the considered cases. The small triangle is a color map key for ease of interpretation.

and $\mathrm{C}_{3 \times 1.5}$ show a difference between the successive profiles above the hub height. The maximum difference is observed between the reconstructed profiles from modes 5 to 10 and from 5 to 25 due to the turbulence kinetic energy contained within these modes.

\subsection{Reynolds stress anisotropy}

To examine the dynamics and energy transfer in the wind turbine arrays with different streamwise and spanwise spacings, a description of the anisotropy upstream and downstream of the wind turbines is presented in Fig. 13. A visualization of the turbulence state is obtained via the color map representing the barycentric map as described in Sect. 2.2. Turbulence anisotropy effectively distinguishes the cases in terms 


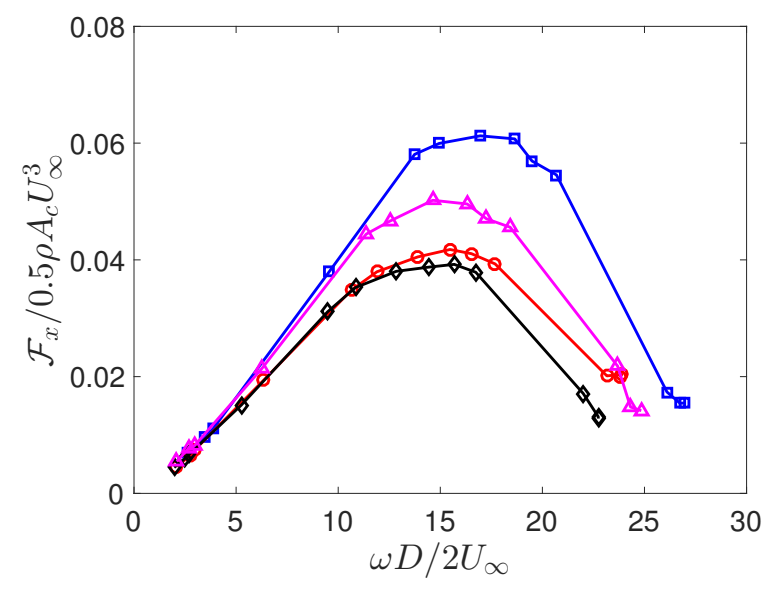

Figure 14. Extracted power of the wind turbine at different angular velocities for four different cases $\mathrm{C}_{6 \times 3}$ (blue $\square$ ), $\mathrm{C}_{3 \times 3}$ (red $\bigcirc$ ), $\mathrm{C}_{3 \times 1.5}$ (black $\left.\diamond\right)$, and $\mathrm{C}_{6 \times 1.5}$ (pink $\triangle$ ).

of wake propagation and wake interaction. The variation in the spacings changes the background turbulence structure. The upstream window of cases $\mathrm{C}_{6 \times 3}$ and $\mathrm{C}_{6 \times 1.5}$ shows that the turbulence field is close to the isotropic limit, especially in hub height region, as a result of the wake recovery occurring under a relatively large spacing distance. Below the bottom tip, these cases show pancake-like turbulence due to the surface effect that appears, deeming the perturbation of the turbines virtually negligible. Near the top of the tip, the flow shows a turbulence of axisymmetric state (between the pancake-like and cigar-like turbulence). With this representation, the spacing variation leads to a changed state of the turbulence and between the developed and developing flow conditions can be discernible. The upstream of case $\mathrm{C}_{3 \times 3}$ shows a pancake-like turbulence state. However, the hub height and bottom tip regions show an isotropic and axisymmetric turbulence, respectively. Upstream of case $\mathrm{C}_{3 \times 1.5}$ exhibits axisymmetric and cigar-like turbulence in most of the upstream domain, although the hub height region continues to show isotropic turbulence.

Past the turbine, the four cases exhibit the turbulence of isotropic state in the hub height region. The top tip region of all four cases shows axisymmetric turbulence, although case $\mathrm{C}_{3 \times 3}$ tends toward cigar-like turbulence. Below hub height, the turbulence is pancake-like and the difference amongst the cases is the covered area, where it is maximum at $\mathrm{C}_{6 \times 3}$ and minimum at $\mathrm{C}_{3 \times 3}$. The longest extension is found in case $\mathrm{C}_{6 \times 3}$ and the lowest in case $\mathrm{C}_{3 \times 3}$. Comparing to $\mathrm{C}_{6 \times 3}$, the change seen in the turbulence states is starker in $\mathrm{C}_{3 \times 3}$ than in $\mathrm{C}_{6 \times 1.5}$, confirming that the impact of reducing streamwise spacing is greater than changing the spanwise spacing. However, the impact of the spanwise spacing is noticeable when $S_{x}=3 D$.

The ability to identify the turbulence structure allows for identification of its influence on subsequent turbines in terms of fatigue loads (Frandsen and Thøgersen, 1999). Further, regions of the flow that are characterized by highly anisotropic turbulence are those in which one is likely to find large-scale, coherent turbulence structures. These structures impart the greatest axial and bending loads onto subsequent turbine rotors, leading to accelerated fatigue and increased operational and maintenance costs for wind farms. In addition, regions of high anisotropy correlate with gradients in the mean flow and turbulence (Hamilton and Cal, 2015). These quantities are of particular interest in wind farm modeling and design. Accordingly, the accurate representation of gradients in wind farm design modeling is a necessary check in accurately representing production of and flux by turbulence kinetic energy, wake interaction, and structural loading on constituent turbines. Finally, the stress tensor invariants, by definition, do not depend on reflection or rotation of the coordinate system, meaning that they are unbiased descriptors for the turbulent flow (Pope, 2000).

\section{Power measurements}

Figure 14 demonstrates the power produced by each turbine, $\mathcal{F}_{x}$, obtained with the torque sensor, versus the angular velocity, $\omega$. The power measurements are normalized by the maximum theoretical power $\frac{1}{2} \rho A_{c} U_{\infty}^{3}$, where $\rho$ is the air density $A_{c}$ is swept area of the turbine rotor $\pi D^{2} / 4$. The angular velocity is normalized by $2 U_{\infty} / D$. It is apparent from the figure that the maximum power is extracted at the normalized angular velocity of $15.8 \pm 1$. The maximum normalized power of 0.062 is harvested at the largest spacing, case $\mathrm{C}_{6 \times 3}$. Fixing the spanwise spacing and decreasing the streamwise spacing reduces the normalized power produced by $33 \%$ for $S_{x}=6 D$ (from case $\mathrm{C}_{6 \times 3}$ to case $\mathrm{C}_{3 \times 3}$ ) and by $22 \%$ for $S_{x}=3 D$ (from case $\mathrm{C}_{3 \times 1.5}$ to case $\mathrm{C}_{6 \times 1.5}$ ). The complementary change in spacing holds the streamwise spacing constant while decreasing the spanwise spacing. In varying the spanwise spacing, the normalized power produced is reduced by $20 \%$ for $S_{z}=3 D$ (from case $\mathrm{C}_{6 \times 3}$ to case $\mathrm{C}_{6 \times 1.5}$ ) and by $6 \%$ for $S_{z}=1.5 D$ (from case $\mathrm{C}_{3 \times 3}$ to case $\mathrm{C}_{3 \times 1.5}$ ). Nilsson et al. (2015) has complementary results to the ones present, in which an increase in power produced is attained in the largest spacing and conversely decreased in the limited spacing case. Increasing the spanwise distance has a less notable effect in comparison to the streamwise spacing.

The trend of the power curves follows that observed in the averaged profiles of the streamwise velocity; see Fig. 7a. Further, they verify the relationship between the power of the turbine with the deficit velocity. The maximum power and velocity are found in case $\mathrm{C}_{6 \times 3}$ and the minimum quantities are noticed in $\mathrm{C}_{3 \times 1.5}$. The smallest variations in the power measurement and main velocity are observed between cases $\mathrm{C}_{3 \times 3}$ and $\mathrm{C}_{3 \times 1.5}$, whereas the largest difference is observed between cases $\mathrm{C}_{6 \times 3}$ and $\mathrm{C}_{3 \times 3}$. Increased longitudinal spacing produces larger energy content in the first few modes and 
establishes the character of the turbulence field of the flow. This is reflected in an increase in power as directly measured via a torque sensing device.

\section{Conclusions}

Insight into the behavior of the flow in a wind turbine array is useful in determining how to highlight the overall power extraction with the variation in spacing between the turbines. The work above quantifies effects of tightly spaced wind turbine configurations on the flow behavior. The findings of this study have a number of important implications, especially regarding the cost of a wind farm or when large areas are not available. Stereoscopic PIV data are used to assess characteristic quantities of the flow field in a wind turbine array with varied streamwise and spanwise spacing. Four cases of different streamwise and spanwise spacings are examined; the streamwise spacing being 6 and $3 D$ and spanwise spacing being 3 and $1.5 D$. The flow fields are analyzed and compared statistically and by snapshot proper orthogonal decomposition.

The streamwise mean velocity and Reynolds shear stress are quantified upstream and downstream of the wind turbine in the considered cases. In the inflow measurement window, higher velocities are observed in cases $\mathrm{C}_{6 \times 3}$ and $\mathrm{C}_{6 \times 1.5}$ compared to the other two cases whose inflows are unrecovered wakes from preceding rows. In contrast, cases $\mathrm{C}_{3 \times 3}$ and $\mathrm{C}_{3 \times 1.5}$ show higher Reynolds shear stress. The notable differences between the cases are found above the top tip and below the bottom tip downstream the turbines, whereas the core of the wakes shows fewer discrepancies. The streamwise and spanwise spacings have a concerted effect on the flow, where the degree of the impact of one change highly depends on the other. This relationship is shown in all statistical quantities discussed here; for example, reducing the streamwise spacing by $50 \%$ leads to increases in the averaged Reynolds shear stress by $16 \%$ when $S_{z}=3 D$. According to current statistical quantities, one can infer that the higher influence of streamwise spacing is shown when the spanwise spacing is $S_{z}=3 D$, and the significant effect of the spanwise spacing is observed when the streamwise spacing is $S_{x}=3 D$. Averaged profiles of the velocity follow the order of higher velocity seen in the contour plots in case $\mathrm{C}_{6 \times 3}$ and lowest velocity in case $\mathrm{C}_{3 \times 1.5}$. The maximum and minimum differences are observed between case $\mathrm{C}_{6 \times 3}$ and case $\mathrm{C}_{3 \times 1.5}$ and case $\mathrm{C}_{3 \times 3}$ and case $C_{3 \times 1.5}$. The result also reveals that the streamwise spacing is more impactful than the spanwise spacing. Spatially averaged profiles of Reynolds shear stress show that the maximum and minimum values occur in cases $\mathrm{C}_{3 \times 3}$ and $\mathrm{C}_{6 \times 1.5}$, respectively.

According to the POD analysis, the upstream measurement plane of the four cases converges faster than the downstream window. Cases $\mathrm{C}_{6 \times 3}$ and $\mathrm{C}_{6 \times 1.5}$ show rapid convergence in cumulative energy content upstream of the turbine, but $\mathrm{C}_{6 \times 3}$ remains behind case $\mathrm{C}_{6 \times 1.5}$ in the wake. The first mode of case $\mathrm{C}_{6 \times 1.5}$ carries the maximum turbulent kinetic energy content compared to the first mode of the other cases. No significant difference in energy content is observed after mode 10 between the four cases. The streamwise-averaged profiles of the Reynolds shear stress are reconstructed by back-projecting coefficients onto the set of eigenfunctions. Low modes are used individually to demonstrate their contributions to the overall flow. Cases $\mathrm{C}_{6 \times 1.5}$ and $\mathrm{C}_{6 \times 3}$ converge to their respective spatially averaged profile faster than the other two cases. The discrepancies in reconstruction are mainly observed in profiles using only the first five modes. The same trend in reconstruction is observed in cases $\mathrm{C}_{3 \times 3}$ and $\mathrm{C}_{3 \times 1.5}$. Reconstructed profiles display the effects of the spacing, where the array of large streamwise spacing reconstructs faster than the other cases due to the coherent structures embedded in the flow.

Based on the Reynolds stress anisotropy tensor and color map visualization, the spacing modifies the anisotropic character of the turbulence. Increased turbine spacing allows the turbulent flow to recover between devices, leading to increasingly isotropic flow incident to the rotors. The hub height region of the wake shows isotropic turbulence regardless of the spacing. The differences in the color map visualization between the downstream locations of the four cases show some structural dependency on the spacing between turbine rotors.

Power production by the turbines is measured directly using a torque sensing system. The power curves follow the same trend as the velocity profiles. The maximum power extracted is at the normalized angular velocity of $15.8 \pm 1$ and it is harvested in case $\mathrm{C}_{6 \times 3}$. The small difference in harvested power is observed between cases $\mathrm{C}_{3 \times 3}$ and $\mathrm{C}_{3 \times 1.5}$. The current work demonstrates that wake statistics and power produced by a wind turbine depend more on streamwise spacing than spanwise spacing. However, the results above pertain only to a fixed inflow direction. In the case in which the bulk flow orientation changes, spacing in both the streamwise and spanwise directions will be important to the optimal power production in a wind turbine array. Continued efforts are required to understand the impact of streamwise and spanwise spacing in infinite array flow under realistic flow conditions, including Coriolis forcing and under different stratification conditions.

Data availability. All data used in this paper are available from the corresponding author upon request.

Competing interests. The authors declare that they have no conflict of interest.

Acknowledgements. Ali acknowledges support from the Higher Committee of Education Development in Iraq. The authors are 
grateful to NSF-ECCS-1032647 for funding this research.

Edited by: Horia Hangan

Reviewed by: three anonymous referees

\section{References}

Ali, N., Aseyev, A. S., and Cal, R. B.: Structure functions, scaling exponents and intermittency in the wake of a wind turbine array, J. Renew. Sustain. Ener., 8, 013304, https://doi.org/10.1063/1.4941782, 2016 a.

Ali, N., Kadum, H. F., and Cal, R. B.: Focused-based multifractal analysis of the wake in a wind turbine array utilizing proper orthogonal decomposition, J. Renew. Sustain. Ener., 8, 063306, 2016b.

Ali, N., Aseyev, A. S., Melius, M. S., Tutkun, M., and Cal, R. B.: Evaluation of Higher Order Moments and Isotropy in the Wake of a Wind Turbine Array, in: Whither Turbulence and Big Data in the 21st Century?, 273-292, Springer, 2017a.

Ali, N., Cortina, G., Hamilton, N., Calaf, M., and Cal, R. B.: Turbulence characteristics of a thermally stratified wind turbine array boundary layer via proper orthogonal decomposition, J. Fluid Mechan., 828, 175-195, 2017b.

Ali, N., Hamilton, N., Cortina, G., Calaf, M., and Cal, R. B.: Anisotropy Stress Invariants of Thermally Stratified Wind Turbine Array Boundary Layer Using Large Eddy Simulations, J. Renew. Sustain. Ener., 10, 2018.

Andersen, S. J., Sørensen, J. N., and Mikkelsen, R.: Simulation of the inherent turbulence and wake interaction inside an infinitely long row of wind turbines, J. Turbulence, 14, 1-24, 2013.

Antonia, R. A., Kim, J., and Browne, L.: Some characteristics of small-scale turbulence in a turbulent duct flow, J. Fluid Mechan., 233, 369-388, 1991.

Archer, C. L., Mirzaeisefat, S., and Lee, S.: Quantifying the sensitivity of wind farm performance to array layout options using large-eddy simulation, Geophys. Res. Lett., 40, 4963-4970, 2013.

Banerjee, S., Krahl, R., Durst, F., and Zenger, C.: Presentation of anisotropy properties of turbulence, invariants versus eigenvalue approaches, J. Turbulence, 8, N32, https://doi.org/10.1080/14685240701506896, 2007.

Barthelmie, R. J. and Jensen, L. E.: Evaluation of wind farm efficiency and wind turbine wakes at the Nysted offshore wind farm, Wind Ener., 13, 573-586, 2010.

Barthelmie, R. J., Frandsen, S. T., Nielsen, M. N., Pryor, S. C., Rethore, P.-E., and Jørgensen, H. E.: Modelling and measurements of power losses and turbulence intensity in wind turbine wakes at Middelgrunden offshore wind farm, Wind Ener., 10, 517-528, 2007.

Barthelmie, R. J., Pryor, S. C., Frandsen, S. T., Hansen, K. S., Schepers, J. G., Rados, K., Schlez, W., Neubert, A., Jensen, L. E., and Neckelmann, S.: Quantifying the impact of wind turbine wakes on power output at offshore wind farms, J. Atmos. Ocean. Technol., 27, 1302-1317, 2010.

Bastine, D., Witha, B., Wächter, M., and Peinke, J.: POD Analysis of a Wind Turbine Wake in a Turbulent Atmospheric Boundary Layer, J. Phys.: Conference Series, 524, 012153, available at: http://stacks.iop.org/1742-6596/524/i=1/a=012153, 2014.
Cal, R. B., Lebrón, J., Castillo, L., Kang, H. S., and Meneveau, C.: Experimental study of the horizontally averaged flow structure in a model wind-turbine array boundary layer, J. Renew. Sustain. Ener., 2, 013106, https://doi.org/10.1063/1.3289735, 2010.

Chamorro, L. P. and Porté-Agel, F.: A wind-tunnel investigation of wind-turbine wakes: Boundary-layer turbulence effects, Bound.Lay. Meteorol., 132, 129-149, 2009.

Chamorro, L. P. and Porté-Agel, F.: Turbulent flow inside and above a wind farm: A wind-tunnel study, Energies, 4, 1916-1936, 2011.

Chamorro, L. P., Arndt, R., and Sotiropoulos, F.: Reynolds number dependence of turbulence statistics in the wake of wind turbines, Wind Ener., 15, 733-742, 2012.

Emory, M. and Iaccarino, G.: Visualizing turbulence anisotropy in the spatial domain with componentality contours, Annual brief, Center for Turbulence Research, 2014.

Frandsen, S. and Thøgersen, M. L.: Integrated fatigue loading for wind turbines in wind farms by combining ambient turbulence and wakes, Wind Eng., 23, 327-339, 1999.

George, W. K.: Lectures in Turbulence for the 21st Century, Chalmers University of Technology, 2013.

Glauser, M. N. and George, W. K.: Orthogonal decomposition of the axisymmetric jet mixing layer including azimuthal dependence, in: Advances in Turbulence, 357-366, Springer, 1987.

Gómez-Elvira, R., Crespo, A., Migoya, E., Manuel, F., and Hernández, J.: Anisotropy of turbulence in wind turbine wakes, J. Wind Eng. Indust. Aerodynam., 93, 797-814, 2005.

González-Longatt, F., Wall, P., and Terzija, V.: Wake effect in wind farm performance: Steady-state and dynamic behavior, Renew. Ener., 39, 329-338, 2012.

Hamilton, N. and Cal, R. B.: Anisotropy of the Reynolds stress tensor in the wakes of wind turbine arrays in Cartesian arrangements with counter-rotating rotors, Phys. Fluids., 27, 015102, https://doi.org/10.1063/1.4903968, 2015.

Hamilton, N., Melius, M., and Cal, R. B.: Wind turbine boundary layer arrays for Cartesian and staggered configurations-Part I, flow field and power measurements, Wind Ener., 18, 277-295, 2015a.

Hamilton, N., Tutkun, M., and Cal, R. B.: Wind turbine boundary layer arrays for Cartesian and staggered configurations: Part II, low-dimensional representations via the proper orthogonal decomposition, Wind Ener., 18, 297-315, 2015b.

Hansen, K. S., Barthelmie, R. J., Jensen, L. E., and Sommer, A.: The impact of turbulence intensity and atmospheric stability on power deficits due to wind turbine wakes at Horns Rev wind farm, Wind Ener., 15, 183-196, 2012.

Kang, H. S. and Meneveau, C.: Direct mechanical torque sensor for model wind turbines, Measure. Sci. Technol., 21, 105206, 2010.

Klipp, C.: Near-surface anisotropic turbulence, in: SPIE Defense, Security, and Sensing., 768 505-768 505, International Society for Optics and Photonics., 2010.

Klipp, C.: Near-surface turbulent temperature variances and anisotropy at multiple scales of motion, in: SPIE Defense, Security, and Sensing., p. 83800G, International Society for Optics and Photonics, 2012.

Krogstad, P. and Torbergsen, L. E.: Invariant analysis of turbulent pipe flow, Flow, turbulence and combustion., 64, 161-181, 2000.

Lumley, J. L.: The structure of inhomogeneous turbulent flows, Atmos. Turbul. Radio Wave Propag., 166-178, 1967. 
Lumley, J. L. and Newman, G. R.: The return to isotropy of homogeneous turbulence, J. Fluid Mechan., 82, 161-178, 1977.

Meyers, J. and Meneveau, C.: Large Eddy simulations of large wind-turbine arrays in the atmospheric boundary layer, AIAA, 827, 2010, 2010.

Meyers, J. and Meneveau, C.: Optimal turbine spacing in fully developed wind farm boundary layers, Wind Ener., 15, 305-317, 2012.

Moin, P. and Moser, R. D.: Characteristic-Eddy decomposition of turbulence in a channel, J. Fluid Mechan., 200, 471-509, 1989.

Nilsson, K., Ivanell, S., Hansen, K. S., Mikkelsen, R., Sørensen, J. N., Breton, S.-P., and Henningson, D.: Large-eddy simulations of the Lillgrund wind farm, Wind Ener., 18, 449-467, 2015.

Pope, S. B.: Turbulent flows, Cambridge University Press, 2000.

Romanic, D., Parvu, D., Refan, M., and Hangan, H.: Wind and tornado climatologies and wind resource modelling for a modern development situated in ?Tornado Alley?, Renew. Ener., 115, 97112, 2018.

Rotta, J.: Statistical theory of nonhomogeneous turbulence, Z. Physik, 131, 51-77, 1951.

Shah, S. and Bou-Zeid, E.: Very-large-scale motions in the atmospheric boundary layer educed by snapshot proper orthogonal decomposition, Bound.-Lay. Meteorol., 153, 355-387, 2014.

Sirovich, L.: Turbulence and the dynamics of coherent structures. Part I: Coherent structures, Q. Appl. Math., 45, 561-571, 1987.

Stevens, R. J.: Dependence of optimal wind turbine spacing on wind farm length, Wind Ener., 19, 651-663, https://doi.org/10.1002/we.1857, 2015.
Stevens, R. J., Gayme, D. F., and Meneveau, C.: Effects of turbine spacing on the power output of extended wind-farms, Wind Ener., 19, 359-370, 2016.

Tutkun, M., Johansson, P. B., and George, W. K.: Three-component vectorial proper orthogonal decomposition of axisymmetric wake behind a disk, AIAA, 46, 1118-1134, 2008.

VerHulst, C. and Meneveau, C.: Large eddy simulation study of the kinetic energy entrainment by energetic turbulent flow structures in large wind farms, Phys. Fluids, 26, 025113, https://doi.org/10.1063/1.4865755, 2014.

Viggiano, B., Gion, M. S., Ali, N., Tutkun, M., and Cal, R. B.: Inverse structure functions in the canonical wind turbine array boundary layer, J. Renew. Sustain. Ener., 8, 053310, https://doi.org/10.1063/1.4966228, 2016.

Wu, Y.-T. and Porté-Agel, F.: Simulation of turbulent flow inside and above wind farms: Model validation and layout effects, Bound.-Lay. Meteorol., 146, 181-205, 2013.

Yang, X., Kang, S., and Sotiropoulos, F.: Computational study and modeling of turbine spacing effects in infinite aligned wind farms, Phys. Fluids (1994-present), 24, 115107, https://doi.org/10.1063/1.4767727, 2012. 